\title{
Energy-Efficient Resource Allocation in Uplink Multiuser Massive MIMO Systems
}

\author{
Ying Hu, ${ }^{1,2}$ Baofeng Ji, ${ }^{3,4}$ Yongming Huang, ${ }^{1}$ Fei Yu, ${ }^{1}$ and Luxi Yang ${ }^{1}$ \\ ${ }^{1}$ School of Information Science and Engineering, Southeast University, Nanjing 210096, China \\ ${ }^{2}$ Institute of Electronics and Information, Jiangsu University of Science and Technology, Zhenjiang, China \\ ${ }^{3}$ Information Engineering College, Henan University of Science and Technology, Luoyang, China \\ ${ }^{4}$ State Key Laboratory of Millimeter Waves, Southeast University, Nanjing 210096, China \\ Correspondence should be addressed to Luxi Yang; lxyang@seu.edu.cn
}

Received 15 July 2014; Revised 7 November 2014; Accepted 12 November 2014

Academic Editor: Xiang Cheng

Copyright ( 2015 Ying Hu et al. This is an open access article distributed under the Creative Commons Attribution License, which permits unrestricted use, distribution, and reproduction in any medium, provided the original work is properly cited.

\begin{abstract}
Energy-efficient communications, namely, green communications, has attracted increasing attention due to energy shortage and greenhouse effect. Motivated by this, we consider the uplink energy-efficient resource allocation in multiuser massive multipleinput multiple-output (MIMO) systems. Specifically, we consider that both the number of antenna arrays at the base station (BS) and the transmit data rate at UE are adjusted adaptively to maximize the energy efficiency. Firstly, we demonstrate the existence of a unique resource allocation solution that is globally optimal by exploiting the properties of objective function. Then we develop an iterative algorithm to solve it. By transforming the originally fractional optimization problem into an equivalent subtractive form using the properties of fractional programming, we develop another efficient iterative resource allocation algorithm. Simulation results have validated the effectiveness of the proposed two algorithms and have shown that both algorithms can fast converge to a near-optimal solution in a small number of iterations.
\end{abstract}

\section{Introduction}

The amount of energy consumption for information and communication technology (ICT) increases dramatically with the exponential growth of service requirement [1], which directly impact on global greenhouse gas emissions. Therefore, as opposed to traditional spectral efficiency resource allocation [2-6], dynamic resource allocation is designed to maximize energy efficiency [7-13]. On the other side, MIMO technology has been proposed to substantially increase the system performance. Basically, the more antennas the transmitter/receiver are equipped with, the more degrees of freedom the propagation channel can provide, the better the performance in terms of data rate or link reliability is [14-17]. Massive MIMO (also known as large-scale antenna systems, very large MIMO, hyper-MIMO, full-dimension MIMO, and ARGOS) makes a clean break with current practice through the use of a large excess of service antennas over active terminals and time-division duplex operation [18]. Recently, multiuser massive MIMO gains more and more attention, where "massive MIMO" usually means the arrays comprising a few hundred antennas simultaneously serving tens of users in the same time-frequency resource [19]. Lower capacity bounds for maximum-ratio combining (MRC), zero-forcing (ZF), and minimum mean-square error (MMSE) detection in massive multiuser MIMO systems are derived in [19]. Whereas it is pointed out in [20] that although MIMO techniques have been shown to be effective in improving capacity and spectral efficiency (SE) of wireless communication systems, energy consumption also increases.

Recently energy-efficient design has emerged as a new trend in wireless communications. The tradeoff between EE (energy efficiency) and SE (spectral efficiency) in downlink multiuser DAS (distributed antenna systems) is addressed in [1]. They first transformed the multicriteria optimization problem with high complexity into a simple single objective 
optimization problem and proposed a novel power allocation algorithm to achieve maximum EE. In [7], the authors consider the energy-efficient power optimization for a general signal cell SISO-OFDM downlink system where subcarriers are allowed to be shared among users. They first proved that OFDMA is optimal for energy-efficient design of the SISO-OFDM system and then turned the nonconvex energyefficient power allocation problem into a quasiconvex optimization problem and proposed an efficient power allocation algorithm. In [9], link adaptive transmission for maximized energy efficiency is addressed. The authors demonstrated the existence of a unique globally optimal link adaptation solution and developed iterative binary search assisted ascent (BSAA) algorithm to obtain it. In [10], the authors address the energy-efficient design of uplink MU-MIMO in a single cell environment. They demonstrated that a unique globally optimal power allocation always exists and gave energy-efficient MU-MIMO power allocation (EMMPA) algorithm to obtain it. In [11], we considered energy-efficient design of resource allocation for a multiuser OFDMA and developed schemes of user selection, rate allocation, and power allocation under QoS requirement to maximize the energy efficiency. In [13], resource allocation for energy-efficient communication in multicell OFDMA downlink network with cooperative base stations is studied. They transformed the considered problem in fractional form into an equivalent optimization problem in subtractive form, which enables the derivation of an efficient iterative resource allocation algorithm.

It is worth mentioning that all the above works only consider energy-efficient resource in single-antenna or fixedbeam OFDM system. There exist few works on the energyefficient design concerning antenna selection for the massive MIMO system. In [21], we consider energy-efficient resource allocation in very large multiuser MIMO single cell systems, in which the effect of large-scale fading is ignored for the sake of simplicity. In [22], an energyefficient iterative resource allocation scheme is proposed for the OFDMA downlink network with a large number of transmit antennas at the BS, which involves multiple-layer iterations and thus is computationally inefficient. Motivated by this, in this paper, we consider uplink energy-efficient resource allocation in multiuser massive MIMO systems. Specifically, in our problem formulation, the number of antenna arrays at BS and the transmit data rate vector at the user are jointly optimized to maximize the energy efficiency, in which the power consumption includes both transmit power and circuit power. Using the energy efficiency lower bound as the optimization criterion, we propose two iterative solutions. We first demonstrate the existence of a unique globally optimal solution by exploiting the properties of objective function; then we develop an iterative algorithm to solve the resource allocation problem. It is proved that the convergence of this iterative algorithm can be guaranteed, but the convergence rate and the performance are sensitive to initial conditions and step length. Herein, we further propose a more efficient iterative algorithm by transforming the considered nonconvex optimization problem in fractional form into an equivalent optimization problem in subtractive form. Its convergence property is also proved. The numerical

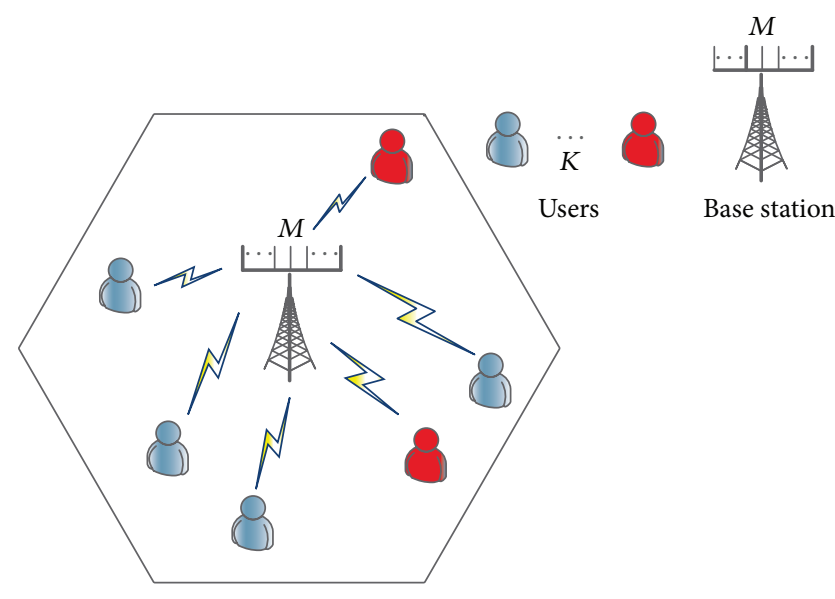

FIgURE 1: The system model.

results show that the proposed algorithms converge to a nearoptimal point with a small number of iterations.

The remainder of this paper is organized as follows. In Section 2, the multiuser massive MIMO system model is described, and the optimization problem for the energyefficient resource allocation is formulated. In Section 3, we provide two iterative algorithms to obtain the optimal solution. Then, we present numerical results in Section 4. Finally, we conclude the paper in Section 5 .

\section{System Model}

As shown in Figure 1, a massive MIMO system consisting of one BS equipped with $M$ antennas and $K$ users each equipped with single antenna is investigated in this paper. The received signal at the BS can be expressed as

$$
\mathbf{Y}=\mathbf{G} \cdot \mathbf{P} \cdot \mathbf{x}+\mathbf{n},
$$

where $\mathbf{Y}=\left[y_{1}, y_{2}, \ldots, y_{M}\right]^{T}$ and []$^{T}$ is the transpose operator. $\mathrm{G}$ is $M \times K$ channel matrix between the $\mathrm{BS}$ and the $K$ users. $\mathbf{P}=\operatorname{diag}\left\{p_{1}, p_{2}, \ldots, p_{K}\right\}$ is the power allocation matrix, where $p_{k}$ is the transmit power of user $k . \mathbf{x}$ is $K \times 1$ transmit signal matrix. $\mathbf{n}$ is $M \times 1$ additive white Gaussian noise (AWGN). Channel matrix $\mathbf{G}$ is given by

$$
\mathbf{G}=\mathbf{H D}^{1 / 2}
$$

where $\mathbf{H}$ is $M \times K$ matrix of fast fading coefficients between the BS and the $K$ users and $\mathbf{D}=\operatorname{diag}\left\{\beta_{1}, \beta_{2}, \ldots, \beta_{K}\right\}$; the component $\beta_{k}$ denotes the large-scale channel factor for user $k$.

As is shown in [19], given the perfect CSI at the receiver when $\mathrm{BS}$ adopts zero-forcing $(\mathrm{ZF})$ receiver, provided that $M \geq K+1$, the achievable uplink rate for the $k$ th user is lower bounded by

$$
r_{k}=\log _{2}\left[1+p_{k}(M-K) \beta_{k}\right] .
$$

We take the noise variance to be 1 , to simplify notation, but without loss of generality. With this convention, $p_{k}$ has the interpretation of normalized "transmit" SNR. 
Correspondingly, the sum data rate is lower bounded by

$$
R=\sum_{k=1}^{K} r_{k}=\sum_{k=1}^{K} \log _{2}\left[1+p_{k}(M-K) \beta_{k}\right] .
$$

The overall transmit power can be written as

$$
P_{T}(\mathbf{R}, M)=\sum_{k=1}^{K} p_{k}=\sum_{k=1}^{K} \frac{2^{r_{k}}-1}{(M-K) \beta_{k}},
$$

where $\mathbf{R}=\left[r_{1}, r_{2}, \ldots, r_{K}\right]^{T}$ is the data rate vector for all users.

Then, the overall power consumption can be expressed as

$$
P(\mathbf{R}, M)=P_{T}(\mathbf{R}, M)+P_{C}(M),
$$

where $P_{C}(M)=M \times p_{c}$ represents the total circuit power consumed by the BS and $p_{c}$ is the circuit power of each antenna which includes the energy consumed by all the circuit blocks along the signal path: analog to digital converter (ADC), digital to analog converter (DAC), frequency synthesizer, mixer, lower noise amplifier (LNA), power amplifier, and baseband DSP [23].

The energy efficiency of interest is then given by

$$
U(\mathbf{R}, M)=\frac{R}{P_{T}(\mathbf{R}, M)+P_{C}(M)} .
$$

Therefore, the resource allocation for the uplink multiuser massive MIMO system can be formulated as the following optimization problem:

$$
\left\{M^{*}, \mathbf{R}^{*}\right\}=\arg \underset{M, \mathbf{R}}{\max _{M}} U(\mathbf{R}, M) .
$$

\section{Energy-Efficient Resource Allocation}

The objective function is a ratio of two functions which generally is a nonconvex function. Next, we will propose two approaches to address this issue. The basic idea of the first approach is to first demonstrate the existence of a unique globally optimal point for the data rate and antenna selection by exploiting the properties of the objective function and then to develop an iterative algorithm to directly solve the optimization problem. While, in the second approach, we first transform the originally fractional optimization problem into an equivalent subtractive form by exploiting the properties of fractional programming, we then develop an efficient iterative resource allocation algorithm to obtain this optimum.

3.1. Direct Optimization Approach. In the following, we first develop Lemma 1 to prove that a unique globally optimal data rate vector and the number of antenna arrays always exist and then further investigate the necessary and sufficient conditions for the globally optimal solution. In particular, we allow $M$ to be a positive real value instead of integer. Then, we can take a derivative with respect to $M$.
TABLE 1: Theorem 2(i).

\begin{tabular}{lc}
\hline$M^{*}$ & Conditions \\
\hline$K+\sqrt{\sum_{k=1}^{K} \frac{2^{r_{k}}-1}{p_{c} \beta_{k}}}$ & $R\left[\sum_{k=1}^{K} \frac{2^{r_{k}}-1}{\beta_{k}}-p_{c}\right] \geq 0$, \\
$\left.\frac{\partial U(\mathbf{R}, M)}{\partial M}\right|_{M=M^{*}}=0$ \\
$R+1$ & $R\left[\sum_{k=1}^{K} \frac{2^{r_{k}}-1}{\beta_{k}}-p_{c}\right]<0$ \\
\hline$R^{*}$ & Conditions \\
\hline$\left.\frac{\partial U(\mathbf{R}, M)}{\partial r_{k}}\right|_{R=R^{*}}=0$ & $P_{T}\left(\mathbf{R}_{k}^{(0)}, M\right)+P_{C}(M)$ \\
$\mathbf{R}_{k}^{(0)}=\left[r_{1}, r_{2}, \ldots, r_{k-1}, 0, r_{k+1}, \ldots, r_{K}\right]^{T}$, & $-R_{k}^{(0)} \bar{P}_{T}^{\prime}(\mathbf{R}, M) \geq 0$ \\
$R_{k}^{(0)}=\sum_{j \neq k} r_{j}\left(\mathbf{R}_{k}^{(0)}, M\right)+P_{C}(M)$ \\
\hline
\end{tabular}

Lemma 1. The energy efficiency function $U(\mathbf{R}, M)$ is (i) strictly quasiconcave (furthermore, $U(\mathbf{R}, M)$ is either strictly decreasing or first strictly increasing and then strictly decreasing) with respect to $M$ for a fixed $\mathbf{R}$ and (ii) strictly quasiconcave (furthermore, $U(\mathbf{R}, M)$ is either strictly decreasing or first strictly increasing and then strictly decreasing) with respect to any $r_{k}$ of $\mathbf{R}$ for a fixed $M$.

Proof. See Appendix A.

Theorem 2. The energy efficiency function $U(\mathbf{R}, M)$ is as follows.

(i) There exists a unique globally optimal number of base station antennas $M^{*}$ for fixed $\mathbf{R}$, where $M^{*}$ is given by Table 1.

(ii) There exists a unique globally optimal transmit rate vector $\mathbf{R}^{*}=\left[r_{1}^{*}, r_{2}^{*}, \ldots, r_{K}^{*}\right]^{T}$ for a fixed $M$, where $r_{k}^{*}$ is given by Table 1 .

Theorem 2 provides the necessary and sufficient conditions for the rate vector $\mathbf{R}^{*}$ and the number of base station antennas $M^{*}$ to be the unique and global optimum. However, it is difficult to directly solve the joint optimization problems. Meanwhile, we can adopt BSAA algorithm [9] to obtain the optimal transmission rate vector $\mathbf{R}^{*}$ for fixed $M$. As a result, we develop an iterative method to search the optimal $\mathbf{R}^{*}$ and $M^{*}$ to maximize $U(\mathbf{R}, M)$. In Algorithm 1, the $U\left(\mathbf{R}^{*}, M^{*}\right)$ is obtained by exhaustive $M^{*}$; therefore the energy efficiency $U(\mathbf{R}, M)$ can converge to the optimal $U\left(\mathbf{R}^{*}, M^{*}\right)$.

In Algorithm 1, BSAA algorithm uses gradient assisted binary search to find the optimal step size $\mu^{*}$ and then adopts $\mathbf{R}=\left[\mathbf{R}+\mu^{*} \nabla U(\mathbf{R})\right]^{+}$to obtain optimal transmission rate vector $\mathbf{R}^{*}$. As shown in flow diagram above, Algorithm 1 gradually increases the transmit rate and the required number of BS antennas to converge to the optimal solution.

3.2. Optimization Approach Based on Problem Transformation. Obviously, the convergence rate and accuracy of Algorithm 1 depend on the value of initialized optimal transmit rate vector and the number of base station antennas as well as the value of step length $\eta$, so we develop the 
(1) $\mathbf{R}=\mathbf{R}_{\mathbf{0}}, M^{*}=M_{0}, U_{\max }=U_{0}$, initialize $\eta>1$

(2) while $U\left(\mathbf{R}^{*}, M^{*}\right)>U_{\max }$

do $U_{\max } \leftarrow U\left(\mathbf{R}^{*}, M^{*}\right)$;

adopt BSAA algorithm to obtain the the optimal transission rate vector $\mathbf{R}^{*}$

$M^{*}=\left\lceil\eta * M^{*}\right\rceil$

Return $U_{\max }, \mathbf{R}^{*}, M^{*}$

Algorithm 1

(1) $\mathbf{R}^{*}=\mathbf{R}_{0}, M^{*}=M_{0}, q^{*}=0$, initialize $\varepsilon=0.01$

(2) while $R^{*}-q^{*}\left[P_{T}\left(\mathbf{R}^{*}, M^{*}\right)+P_{C}\left(M^{*}\right)\right]>\varepsilon$

$\operatorname{do} q^{*} \leftarrow \frac{R\left(r^{*}\right)}{\left[P_{T}\left(\mathbf{R}^{*}, M\right)+P_{C}\left(M^{*}\right)\right]}$

adopt formula (13) above obtain rate allocation

adopt formula (12) above obtain the number of base station antennas

Return $q^{*}, r^{*}, M^{*}$

Algorithm 2

indirect algorithm to search locally optimal $\mathbf{R}^{*}$ and $M^{*}$ to maximize $U(\mathbf{R}, M)$. The fractional objective function in (7) can be classified as a nonlinear fractional program [24]. We can get the following theorem from theorem in [24].

Theorem 3. Consider

$$
q^{*}=\frac{R^{*}}{P_{T}\left(\mathbf{R}^{*}, M^{*}\right)+P_{C}\left(M^{*}\right)}=\underbrace{\max }_{M, \mathbf{R}} \frac{R}{P_{T}(\mathbf{R}, M)+P_{C}(M)}
$$

if and only if

$$
\begin{array}{rl}
\underbrace{\max }_{M, \mathbf{R}} & R-q^{*}\left[P_{T}(\mathbf{R}, M)+P_{C}(M)\right] \\
& =R^{*}-q^{*}\left[P_{T}\left(\mathbf{R}^{*}, M^{*}\right)+P_{C}\left(M^{*}\right)\right] \\
& =0,
\end{array}
$$

for $R \geq 0$ and $P_{T}(\mathbf{R}, M)+P_{C}(M)>0$.

According to Theorem 3, for any optimization problem with an objective function in fractional form, there always exists an equivalent objective function in subtractive form. As a result, we can focus on the equivalent objective function:

$$
F(q)=\underbrace{\max _{1}}_{M, \mathbf{R}} R-q\left[P_{T}(\mathbf{R}, M)+P_{C}(M)\right]
$$

in the rest of the paper.

The problem above is now joint concave with respect to all variables if $2 \sum_{k=1}^{K}\left(2^{r_{k}}-1\right)-2^{r_{k}} \geq 0$ (cf. Appendix B).
Using standard optimization technique, the number of base station antennas is

$M^{*}$

$$
=\left\lceil\frac{(K / q \ln 2)+\sqrt{(K / q \ln 2)^{2}-4 p_{c} \sum_{k=1}^{K}\left(1 / \beta_{k}\right)}}{2 p_{c}}+K\right\rceil .
$$

And the rate allocation is

$$
r_{k}^{*}=\log _{2}\left[\frac{(M-K) \beta_{k}}{q \ln 2}\right] .
$$

Therefore, we develop an iterative algorithm to search the optimal $\mathbf{R}, M^{*}$, and $q^{*}$ to maximize function $f$.

Note that this iterative algorithm guarantees convergence. Please refer to [21] for a proof of convergence. As shown in flow diagram above, with the increment of the value of $q$, Algorithm 2 gradually decreases the transmit rate and the required number of BS antennas to converge to the optimal solution.

\section{Simulations}

In this section, we provide the simulation results to evaluate the performance of our proposed algorithms varying with the number of users. Moreover, we also provide the simulation results to evaluate the energy-efficiency performance varying with the number of iterations and the number of BS antennas. In order to reduce the computational complexity of optimal algorithm, throughout the simulations, we assume that the users are divided into three groups according to their distance to the base station; that is, $\beta=[1,0.8,0.5]$. 


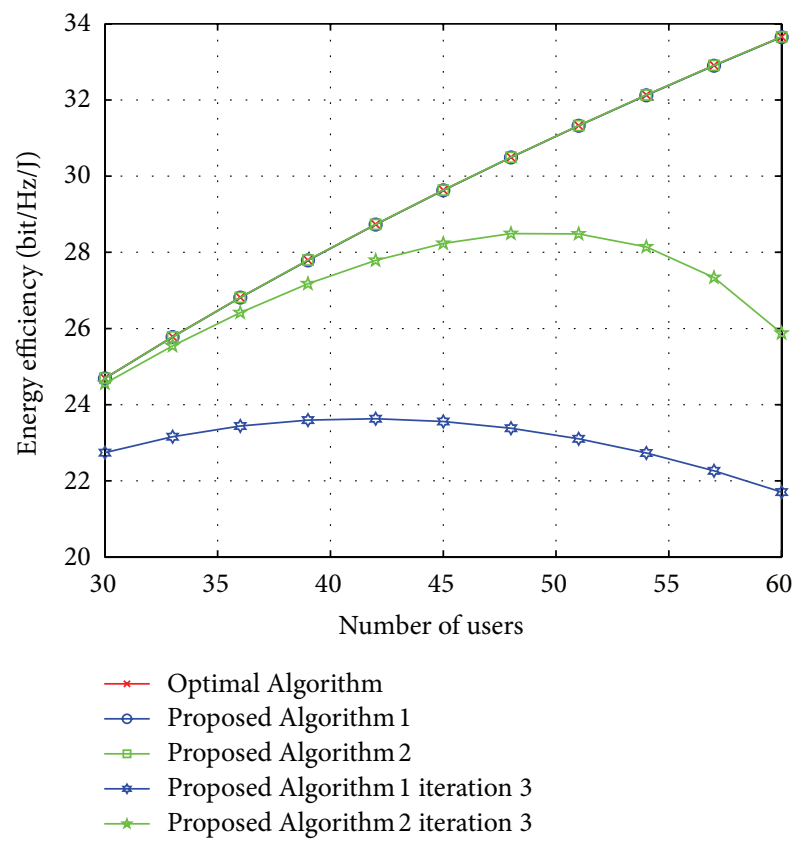

FIGURE 2: Energy efficiency versus number of users.

Meanwhile, we consider a static circuit power consumption $p_{c}=10 \mathrm{mw}$ and use an initialized optimal transmit rate vector $\mathbf{R}_{0}=[0.1, \ldots, 0.1]^{T}, \eta=1.02$. To examine the gap of the performance of the proposed algorithm to the globally optimal solution, we use exhaustive search method to obtain the optimal performance. The "proposed Algorithm 1 iteration 3" means that we adopt Algorithm 1 with a given number of iterations of 3 , and the same to the proposed Algorithm 2 iteration 3.

Figure 2 shows the energy efficiency of the proposed algorithms versus the number of users. As clearly seen, the energy efficiency increases with the number of users. It is also shown that the performance of both proposed algorithms is very close to the optimum. When the number of iterations is limited to 3, the results show that Algorithm 2 achieves a near-convergence performance when the user number is less than 35, while the gap between Algorithm 2 and optimal solution increases with the user number growing. This is because the number of iterations will increase with the user number growing for achieving the optimal solution. In comparison, the performance of Algorithm 1 with 3 iterations exhibits a large gap to its converged solution.

Figure 3 depicts the optimal number of base station antennas $M^{*}$ versus the number of users. As expected, the number of base station antennas required in our resource allocation algorithms increases with the number of users. The convergency performances of our proposed two algorithms are both very close to the global optimum. While the number of iterations is fixed at 3, the performance of two algorithms is quite different. The required number of BS antennas of Algorithm 2 increases with the number of users. But that of Algorithm 1 stays unchanged even though more users are

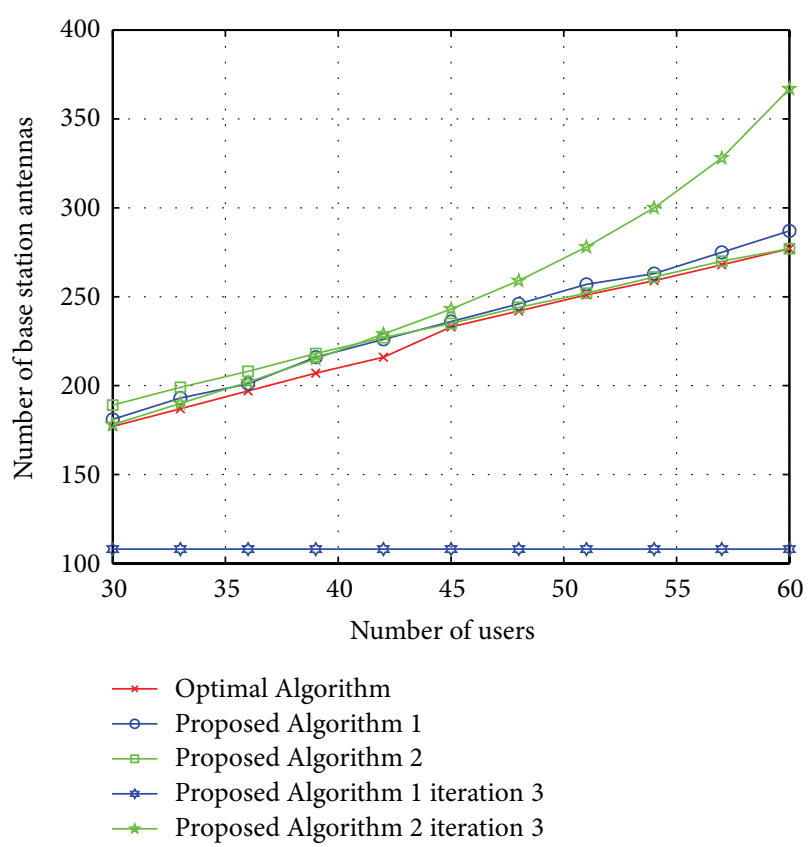

FIGURE 3: Number of base station antennas versus number of users.

deployed. This is because the required number of BS antennas of Algorithm 1 is updated by $M^{*}=\left\lceil\eta * M^{*}\right\rceil$. When the value of $\eta$ is small, the update speed is very slow. Whereas the required number of BS antennas of Algorithm 2 is updated by $M^{*}=\left\lceil\left((K / q \ln 2)+\sqrt{(K / q \ln 2)^{2}-4 p_{c} \sum_{k=1}^{K}\left(1 / \beta_{k}\right)}\right) / 2 p_{c}+\right.$ $K\rceil$, the required number of $\mathrm{BS}$ antennas of Algorithm 2 increases with the number of users growing. Meanwhile, when the value of $q$ is smaller, the required number of BS antennas is larger. The results also show when serving tens of users, the optimal number of base station antennas needs a hundred or a few hundred.

Figure 4 illustrates the overall transmit power versus the number of users. As clearly seen, the overall transmit power increases with the number of users. Furthermore, it is also shown that the performance of both proposed algorithms is close to the optimum. Whereas the overall transmit power of the proposed algorithms with a given number of iterations is different, when iteration number is fixed at 3, the transmit power of Algorithm 2 is larger than the optimal value, but that of Algorithm 1 is smaller. This is because the transmit rate of Algorithm 2 is updated by $r_{k}^{*}=\log _{2}\left[(M-K) \beta_{k} / q \ln 2\right]$; when the value of $q$ is smaller than the optimal value, the transmit rate is larger, so the transmit power is larger than the optimal value. Whereas the transmit rate of Algorithm 1 is updated by BSAA algorithm when the required number of BS antennas is fixed, the transmit power of Algorithm 1 is smaller than the optimal value.

Figure 5 depicts the spectral efficiency versus the number of users. It is seen that the trend of the spectral efficiency versus the number of users is the same as the optimal number of base station antennas $M^{*}$ versus the number of users. It also can be seen that Algorithm 2 iteration 


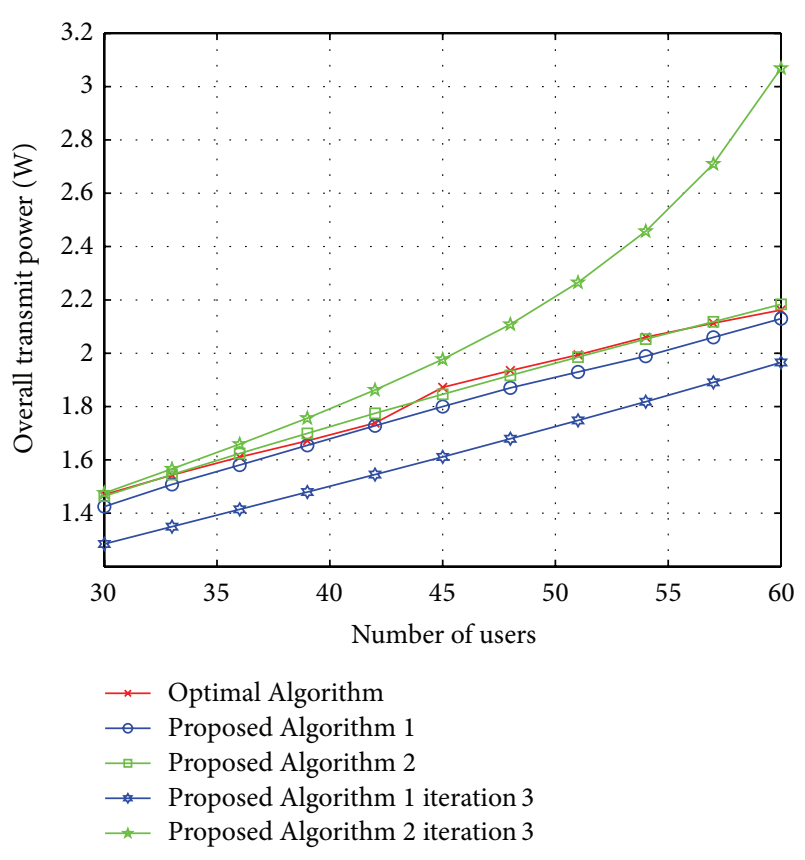

Figure 4: Overall transmit power versus number of users.

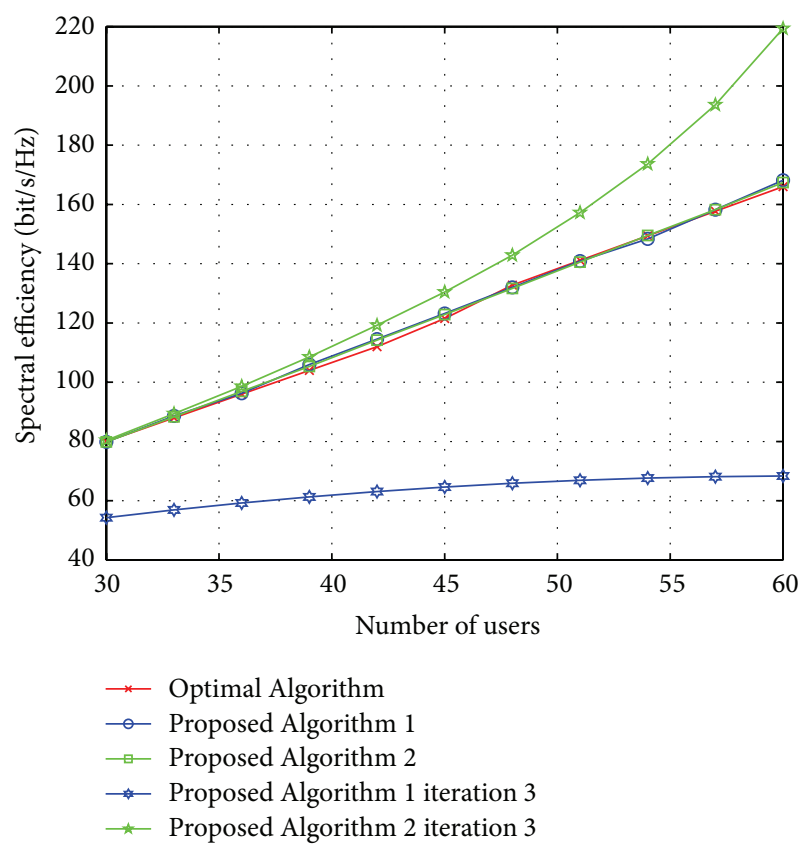

FIgURE 5: Spectral efficiency versus number of users.

3 attains even higher spectral efficiency than the optimal result. This is because the transmit rate of Algorithm 2 is updated by $r_{k}^{*}=\log _{2}\left[(M-K) \beta_{k} / q \ln 2\right]$; when the value of $q$ is smaller than the optimal value, the transmit rate is larger than the optimal value. In a word, when the iteration number is fixed at 3 , the performances of two algorithms are quite different. This is because the convergence manner of

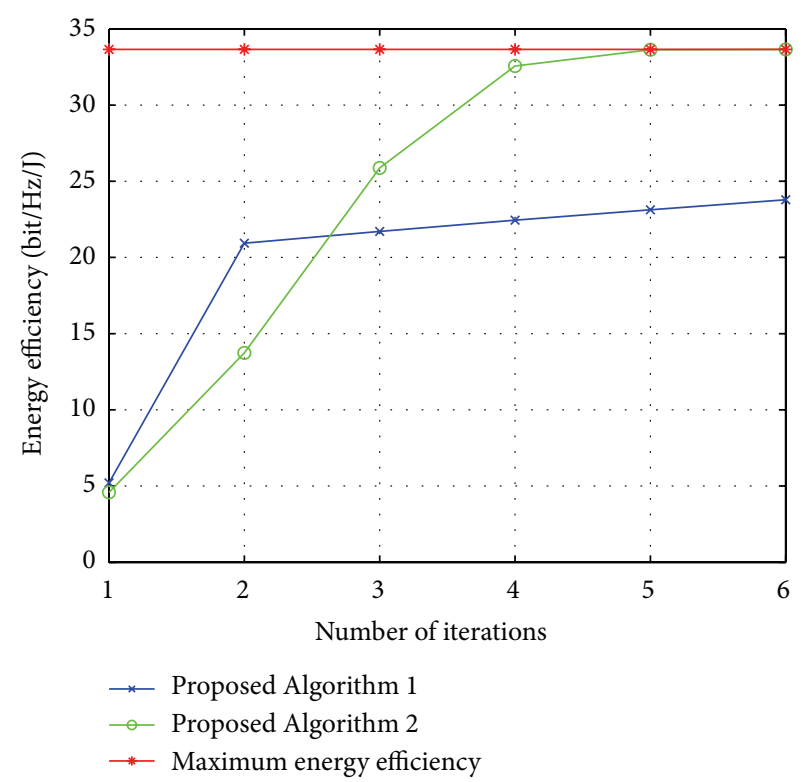

FIGURE 6: Energy efficiency versus the number of iterations.

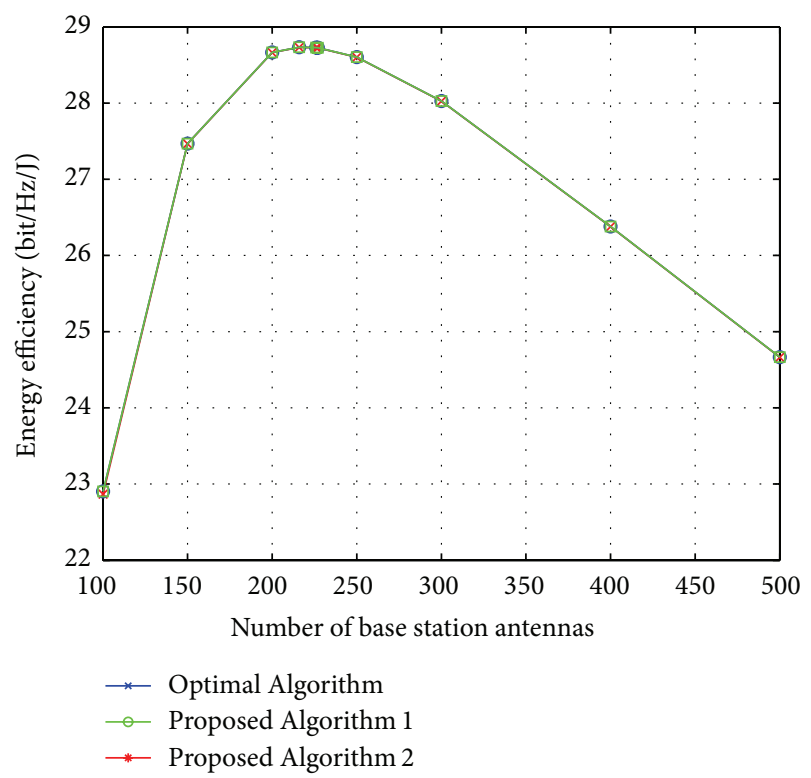

FIGURE 7: Energy efficiency versus number of base station antennas.

the two algorithms is quite different. Algorithm 1 gradually increases the transmit rate and the required number of BS antennas to converge to the optimal solution, whereas Algorithm 2 gradually decreases the transmit rate and the required number of $\mathrm{BS}$ antennas to converge to the optimal solution.

Figure 6 shows the energy efficiency versus the number of iterations for $K=60$. It is observed that the proposed Algorithm 2 achieves $97 \%$ of the convergency performance in 4 iterations, while the proposed Algorithm 2 obtains $71 \%$ of the convergency performance with 6 iterations. In a word, 


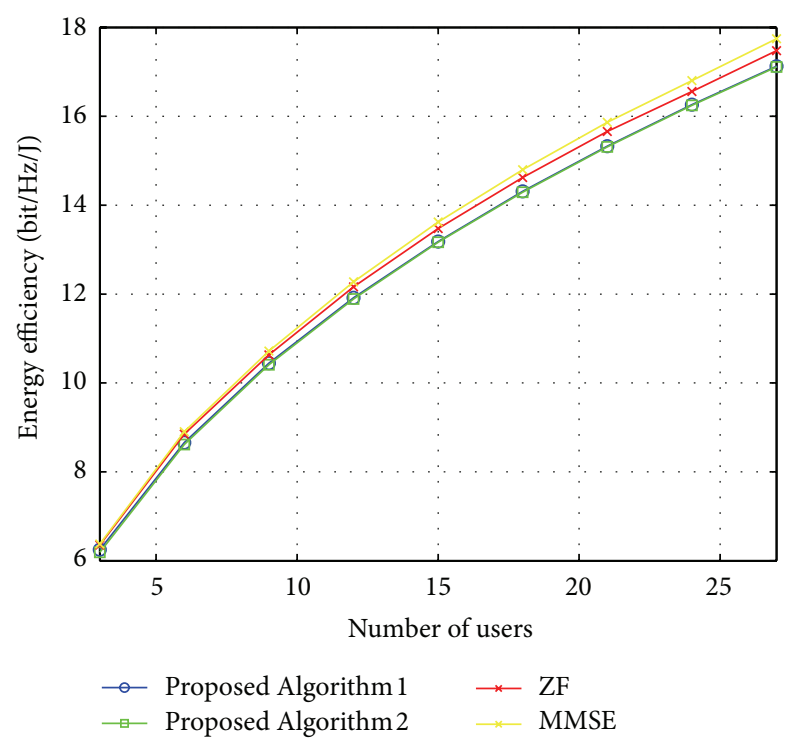

FIGURE 8: Energy efficiency versus number of users.

most of the potential energy efficiency can be achieved with a few number of iterations.

Figure 7 shows the energy efficiency versus optimal number of base station antennas $M^{*}$ for $K=42$. It is observed that there exist a unique globally optimal number of base station antennas $M^{*}$ to maximize energy efficiency.

Note that we evaluate the energy efficiency via expression (8) in the above, where the fast fading coefficient is ignored. To investigate the accuracy of this expression under real channel environment and further to examine our proposed algorithms with practical receivers, Figure 8 shows the energy efficiency of the proposed algorithms versus the number of users, where fast fading channels and linear ZF/MMSE receivers [12] are considered. The results show that the performance of the proposed two algorithms evaluated with (8) is very close to that achieved with linear receivers under fading channels.

\section{Conclusion}

In this paper, we have investigated uplink energy-efficient resource allocation in multiuser massive MIMO systems. Our goal is to jointly optimize rate allocation and the number of antenna arrays at BS, in order to make the performance measure in terms of throughput per Joule maximizing, in which the power consumption includes both transmit power and circuit power. We first demonstrate the existence of a unique globally optimal solution by exploiting the properties of objective function and then develop an iterative algorithm to solve the resource allocation problem. Furthermore, we propose a more efficient iterative algorithm by transforming the originally nonconvex optimization problem in fractional form into an equivalent optimization problem in subtractive form. It is proved that the proposed two algorithms guarantee convergence. And the numerical results show that the proposed algorithms converge to a near-optimal point with a small number of iterations.

\section{Appendices}

\section{A. Proof of Lemma 1}

(i) For a fixed $\mathbf{R}$, denote the upper contour sets of $U(\mathbf{R}, M)$ by $S_{\alpha}=\{M \geq K+1 \mid U(\mathbf{R}, M) \geq \alpha\}$.

According to proposition C.9 of [25], $U(\mathbf{R}, M)$ is strictly quasiconcave if and only if $S_{\alpha}$ is strictly convex for any real number $\alpha$. When $\alpha \leq 0$, no points exist on the contour $U(\mathbf{R}, M)=\alpha$. Hence, $S_{\alpha}$ is strictly convex when $\alpha \leq 0$. Then, we investigate the case when $\alpha>0, S_{\alpha}$ is equivalent to

$$
S_{\alpha}=\left\{M \geq K+1 \mid \alpha P_{T}(\mathbf{R}, M)+\alpha P_{C}(M)-R \leq 0\right\} .
$$

Clearly $P_{T}(\mathbf{R}, M)$ and $P_{C}(M)$ are strictly convex in $M$. Therefore, $S_{\alpha}$ is strictly convex and we have the strict quasiconcavity of $U(\mathbf{R}, M)$.

The partial derivative of $U(\mathbf{R}, M)$ with respect to $M$ is

$$
\begin{aligned}
\frac{\partial U(\mathbf{R}, M)}{\partial M} & =\frac{-R\left[P_{T}^{\prime}(\mathbf{R}, M)+P_{C}^{\prime}(M)\right]}{\left[P_{T}(\mathbf{R}, M)+P_{C}(M)\right]^{2}} \\
& \triangleq \frac{\phi(\mathbf{R}, M)}{\left[P_{T}(\mathbf{R}, M)+P_{C}(M)\right]^{2}},
\end{aligned}
$$

where $P_{T}^{\prime}(\mathbf{R}, M)$ is the first partial derivative of $P_{T}(\mathbf{R}, M)$ with respect to $M$ and $P_{C}^{\prime}(M)$ is the first partial derivative of $P_{C}(M)$ with respect to $M$. According to Lemma 1, if $M^{*}$ exists such that $\left.(\partial U(\mathbf{R}, M) / \partial M)\right|_{M=M^{*}}=0$, it is unique; that is, there is a $M^{*}$ such that $\phi\left(\mathbf{R}, M^{*}\right)=0$; it is unique. In the following, we investigate the conditions when $M^{*}$ exists.

The derivative of $\phi(\mathbf{R}, M)$ with respect to $M$ is $\phi^{\prime}(\mathbf{R}, M)=$ $-R P_{T}^{\prime \prime}(\mathbf{R}, M)<0$, where $P_{T}^{\prime \prime}(\mathbf{R}, M)$ is the second partial derivative of $P_{T}(\mathbf{R}, M)$ with respect to $M$. Hence, $\phi(\mathbf{R}, M)$ is strictly decreasing. According to the L'Hopital's rule, it is easy to show that

$$
\begin{aligned}
\lim _{M \rightarrow \infty} \phi(\mathbf{R}, M) & =\lim _{M \rightarrow \infty}\left\{-R\left[P_{T}^{\prime}(\mathbf{R}, M)+P_{C}^{\prime}(M)\right]\right\} \\
& =\lim _{M \rightarrow \infty}\left\{\frac{-R\left[P_{T}^{\prime}(\mathbf{R}, M)+P_{C}^{\prime}(M)\right]}{M} M\right\} \\
& =\lim _{M \rightarrow \infty}\left[\frac{-R P_{T}^{\prime \prime}(\mathbf{R}, M)}{1} M\right]<0 .
\end{aligned}
$$

Because of the $M \geq K+1$, where $K$ is the number of users,

$$
\begin{aligned}
\lim _{M \rightarrow K+1} \phi(\mathbf{R}, M) & =\lim _{M \rightarrow K+1}\left\{-R\left[P_{T}^{\prime}(\mathbf{R}, M)+P_{T}^{\prime}(M)\right]\right\} \\
& =-R\left[-\sum_{k=1}^{K} \frac{2^{r_{k}}-1}{\beta_{k}}+p_{c}\right] \\
& =R\left[\sum_{k=1}^{K} \frac{2^{r_{k}}-1}{\beta_{k}}-p_{c}\right] .
\end{aligned}
$$


(1) When $R\left[\sum_{k=1}^{K}\left(\left(2^{r_{k}}-1\right) / \beta_{k}\right)-p_{c}\right] \geq 0$, $\lim _{M \rightarrow K+1} \phi(\mathbf{R}, M) \geq 0$. We can see that $M^{*}$ exists and $U(\mathbf{R}, M)$ is first strictly increasing and then strictly decreasing in $M$.

(2) When $R\left[\sum_{k=1}^{K}\left(\left(2^{r_{k}}-1\right) / \beta_{k}\right)-p_{c}\right]<0$, $\lim _{M \rightarrow K+1} \phi(\mathbf{R}, M)<0$. We can see that $U(\mathbf{R}, M)$ is always strictly decreasing in $M$. Hence, $U(\mathbf{R}, M)$ is maximized at $M=K+1$.

(ii) The quasiconcavity of $U(\mathbf{R}, M)$ with respect to $r_{k}$ is the same as the quasiconcavity of $U(\mathbf{R}, M)$ with respect to $M$.

The partial derivative of $U(\mathbf{R}, M)$ with respect to $r_{k}$ is

$$
\begin{aligned}
\frac{\partial U(\mathbf{R}, M)}{\partial r_{k}} & =\frac{R^{\prime}\left[P_{T}(\mathbf{R}, M)+P_{C}(M)\right]-R \bar{P}_{T}^{\prime}(\mathbf{R}, M)}{\left[P_{T}(\mathbf{R}, M)+P_{C}(M)\right]^{2}} \\
& \triangleq \frac{\varphi\left(r_{k}, M\right)}{\left[P_{T}(\mathbf{R}, M)+P_{C}(M)\right]^{2}},
\end{aligned}
$$

where $\bar{P}_{T}^{\prime}(\mathbf{R}, M)$ is the first partial derivative of $P_{T}(\mathbf{R}, M)$ with respect to $r_{k}$. According to Lemma 1 , if $r_{k}$ exists such that $\left.\left(\partial U(\mathbf{R}, M) / \partial r_{k}\right)\right|_{r_{k}=r_{k}^{*}}=0$, it is unique; that is, there is a $r_{k}^{*}$ such that $\varphi\left(r_{k}^{*}, M\right)=0$; it is unique. In the following, we investigate the conditions when $r_{k}^{*}$ exists.

The derivative of $\varphi\left(r_{k}, M\right)$ with respect to $r_{k}$ is

$$
\varphi^{\prime}\left(r_{k}, M\right)=-R \bar{P}_{T}^{\prime \prime}(\mathbf{R}, M)<0,
$$

where $\bar{P}_{T}^{\prime \prime}(\mathbf{R}, M)$ is the second partial derivative of $P_{T}(\mathbf{R}, M)$ with respect to $r_{k}$. Hence, $\varphi\left(r_{k}, M\right)$ is strictly decreasing. According to the L'Hopital's rule, it is easy to show that

$$
\begin{aligned}
& \lim _{r_{k} \rightarrow \infty} \varphi\left(r_{k}, M\right) \\
& \quad=\lim _{r_{k} \rightarrow \infty}\left\{\left[P_{T}(\mathbf{R}, M)+P_{C}(M)\right]-R \bar{P}_{T}^{\prime}(\mathbf{R}, M)\right\} \\
& \quad=\lim _{r_{k} \rightarrow \infty}\left\{\frac{\left[P_{T}(\mathbf{R}, M)+P_{C}(M)\right]-R \bar{P}_{T}^{\prime}(\mathbf{R}, M)}{r_{k}} r_{k}\right\} \\
& \quad=\lim _{r_{k} \rightarrow \infty}\left[\frac{-R \bar{P}_{T}^{\prime \prime}(\mathbf{R}, M)}{1} r_{k}\right]<0, \\
& \lim _{r_{k} \rightarrow 0} \phi\left(r_{k}, M\right) \\
& \quad=\lim _{r_{k} \rightarrow 0}\left\{\left[P_{T}(\mathbf{R}, M)+P_{C}(M)\right]-R \bar{P}_{T}^{\prime}(\mathbf{R}, M)\right\} \\
& \quad=P_{T}\left(\mathbf{R}_{k}^{(0)}, M\right)+P_{C}(M)-R_{k}^{(0)} \bar{P}_{T}^{\prime}\left(\mathbf{R}_{k}^{(0)}, M\right),
\end{aligned}
$$

where $\mathbf{R}_{k}^{(0)}=\left[r_{1}, r_{2}, \ldots, r_{k-1}, 0, r_{k+1}, \ldots, r_{K}\right]^{T}$ and $\mathbf{R}_{k}^{(0)}=$ $\sum_{j \neq k} r_{j}$.

(1) When $P_{T}\left(\mathbf{R}_{k}^{(0)}, M\right)+P_{C}(M)-R_{k}^{(0)} \bar{P}_{T}^{\prime}(\mathbf{R}, M) \geq 0$, $\lim _{r_{k} \rightarrow 0} \phi\left(r_{k}, M\right) \geq 0$. We see that $r_{k}^{*}$ exists and $U(\mathbf{R}, M)$ is first strictly increasing and then strictly decreasing in $r_{k}$.
(2) When $P_{T}\left(\mathbf{R}_{k}^{(0)}, M\right)+P_{C}(M)-R_{k}^{(0)} \bar{P}_{T}^{\prime}(\mathbf{R}, M)<0$, $\lim _{r_{k} \rightarrow 0} \phi\left(r_{k}, M\right)<0$. We see that $U(\mathbf{R}, M)$ is always decreasing in $r_{k}$. Hence, $U(\mathbf{R}, M)$ is maximized at $r_{k}=$ 0 .

Then, Lemma 1 is readily obtained.

\section{B. Proof of the Concavity of the Problem in (11)}

Without loss of generality, we define function

$$
\begin{aligned}
f & =R-q\left[P_{T}(\mathbf{R}, M)+P_{C}(M)\right] \\
& =\sum_{k=1}^{K} r_{k}-q\left[\sum_{k=1}^{K} \frac{2^{r_{k}}-1}{(M-K) \beta_{k}}+M p_{c}\right] .
\end{aligned}
$$

The Hessian matrix is given by

$$
\begin{aligned}
& H(f)=\left(\begin{array}{cc}
-q K \frac{2^{r_{k}}(\ln 2)^{2}}{(M-K) \beta_{k}} & q K \frac{2^{r_{k}} \ln 2}{(M-K)^{2} \beta_{k}} \\
q K \frac{2^{r_{k}} \ln 2}{(M-K)^{2} \beta_{k}} & -2 q K \frac{2^{r_{k}}-1}{(M-K)^{3} \beta_{k}}
\end{array}\right), \\
& \operatorname{def}(f)=q^{2} \frac{2^{r_{k}}(\ln 2)^{2}}{(M-K)^{4}}\left[\frac{2 \sum_{k=1}^{K}\left(2^{r_{k}}-1\right)-2^{r_{k}}}{\beta_{k}^{2}}\right] .
\end{aligned}
$$

As a matter of fact, the function $f$ is, in general, not concave, which means that a strictly joint optimal $f$ with respect to the complete parameter set $(\mathbf{R}, M)$ cannot be obtained via a convex optimization technique. Note that if $2 \sum_{k=1}^{K}\left(2^{r_{k}}-1\right)-2^{r_{k}} \geq 0, H(f)$ is a negative semidefinite matrix. Therefore, $f$ is jointly concave with respect to $\mathbf{R}$ and $M$. On the other hand, the objective function is jointly concave with respect to $\mathbf{R}$ and $M$.

\section{Conflict of Interests}

The authors declare that there is no conflict of interests regarding the publication of this paper.

\section{Acknowledgments}

This work was supported by the National Science and Technology Major Project of China under Grant 2012ZX03004005-003, National Natural Science Foundation of China under Grants 61201172, 61271018, 61071113, and 61201176, Joint Funds of the National Natural Science Foundation of China under Grant U1404615, Open Funds of State Key Laboratory of Millimeter Waves under Grant K201504, the Research Project of Jiangsu Province under Grants BK20130019 and BE2012167, and the Program for New Century Excellent Talents in University under Grant NCET-11-0088. 


\section{References}

[1] C. He, B. Sheng, P. Zhu, and X. You, "Energy efficiency and spectral efficiency tradeoff in downlink distributed antenna systems," IEEE Wireless Communications Letters, vol. 1, no. 3, pp. 153-156, 2012.

[2] C.-X. Zhong and L.-X. Yang, "User scheduling and power allocation for the downlink of MIMO systems based on limited feedback," in Proceedings of the 1st International Workshop on Cross Layer Design (IWCLD '07), pp. 10-13, Jinan, China, September 2007.

[3] Y. Huang, G. Zheng, M. Bengtsson, K.-K. Wong, L. Yang, and B. Ottersten, "Distributed multicell beamforming with limited intercell coordination," IEEE Transactions on Signal Processing, vol. 59, no. 2, pp. 728-738, 2011.

[4] S. He, Y. Huang, L. Yang, A. Nallanathan, and P. Liu, "A multicell beamforming design by uplink-downlink max-min SINR duality," IEEE Transactions on Wireless Communications, vol. 11, no. 8, pp. 2858-2867, 2012.

[5] L. Chen, X. Wang, and G. He, "Dynamic resource allocation with precoding and joint coding scheme for limited feedbackbased wireless multi-antenna multicast system," IET Communications, vol. 7, no. 5, pp. 397-407, 2013.

[6] S. P. Shariatpanahi, B. H. Khalaj, and H. Shah-Mansouri, "Power allocation scheme in time division multiple access distributed multiple-input multiple-output interference channels," IET Communications, vol. 7, no. 5, pp. 391-396, 2013.

[7] Q. Shi, W. Xu, D. Li, Y. Wang, X. Gu, and W. Li, “On the energy efficiency optimality of OFDMA for SISO-OFDM downlink system," IEEE Communications Letters, vol. 17, no. 3, pp. 541544, 2013.

[8] G. W. Miao, G. Y. Li, S. Q. Zhang, Y. Chen, and S. G. Xu, "Energy-efficient resource allocation in OFDMA networks," IEEE Transactions on Communications, vol. 60, no. 12, pp. 37673778, 2012.

[9] G. Miao, N. Himayat, and G. Y. Li, "Energy-efficient link adaptation in frequency-selective channels," IEEE Transactions on Communications, vol. 58, no. 2, pp. 545-554, 2010.

[10] G. Miao, "Energy-efficient uplink multi-user MIMO," IEEE Transactions on Wireless Communications, vol. 12, no. 5, pp. 2302-2313, 2013.

[11] Y. Hu, Y. Huang, L. Yang, and J. Zhou, "Energy-efficient resource allocation in multi-user OFDMA systems," in Proceedings of the International Conference on Wireless Communications and Signal Processing (WCSP '11), pp. 1-5, Nanjing, China, November 2011.

[12] B. F. Ji, K. Song, J. Zhu, and W. K. Li, "Efficient MAC protocol design and performance analysis for dense WLANs," Wireless Networks, vol. 20, no. 8, pp. 2237-2254, 2014.

[13] D. W. K. Ng, E. S. Lo, and R. Schober, "Energy-efficient resource allocation in multi-cell OFDMA systems with limited backhaul capacity," IEEE Transactions on Wireless Communications, vol. 11, no. 10, pp. 3618-3631, 2012.

[14] F. Rusek, D. Persson, B. K. Lau et al., "Scaling up MIMO: opportunities and challenges with very large arrays," IEEE Signal Processing Magazine, vol. 30, no. 1, pp. 40-60, 2013.

[15] X. Cheng, B. Yu, L. Yang et al., "Communicating in the real world: 3D MIMO," IEEE Wireless Communications Magazine, vol. 21, no. 4, pp. 136-144, 2014.

[16] X. Cheng, C.-X. Wang, H. Wang et al., "Cooperative MIMO channel modeling and multi-link spatial correlation properties,"
IEEE Journal on Selected Areas in Communications, vol. 30, no. 2, pp. 388-396, 2012.

[17] B. Ji, J. Zhu, K. Song, Y. Huang, and L. Yang, "Performance analysis of femtocells network with co-channel interference," Signal Processing, vol. 100, pp. 32-41, 2014.

[18] E. G. Larsson, O. Edfors, F. Tufvesson, and T. L. Marzetta, "Massive MIMO for next generation wireless systems," IEEE Communications Magazine, vol. 52, no. 2, pp. 186-195, 2014.

[19] H. Q. Ngo, E. G. Larsson, and T. L. Marzetta, "Energy and spectral efficiency of very large multiuser MIMO systems," IEEE Transactions on Communications, vol. 61, no. 4, pp. 1436-1449, 2013.

[20] G. Y. Li, Z. Xu, C. Xiong et al., "Energy-efficient wireless communications: tutorial, survey, and open issues," IEEE Wireless Communications, vol. 18, no. 6, pp. 28-34, 2011.

[21] Y. Hu, B. Ji, Y. Huang, F. Yu, and L. Yang, "Energy-efficiency resource allocation of very large multi-user MIMO systems," Wireless Networks, vol. 20, no. 6, pp. 1421-1430, 2014.

[22] D. W. K. Ng, E. S. Lo, and R. Schober, "Energy-efficient resource allocation in OFDMA systems with large numbers of base station antennas," IEEE Transactions on Wireless Communications, vol. 11, no. 9, pp. 3292-3304, 2012.

[23] S. Cui, A. J. Goldsmith, and A. Bahai, "Energy-efficiency of MIMO and cooperative MIMO techniques in sensor networks," IEEE Journal on Selected Areas in Communications, vol. 22, no. 6, pp. 1089-1098, 2004.

[24] W. Dinkelbach, "On nonlinear fractional programming," Management Science, vol. 13, pp. 492-498, 1967.

[25] E. Wolfstetter, Topics in Microeconomics: Industrial Organization, Auctions, and Incentives, Cambridge University Press, Cambridge, UK, 1999. 

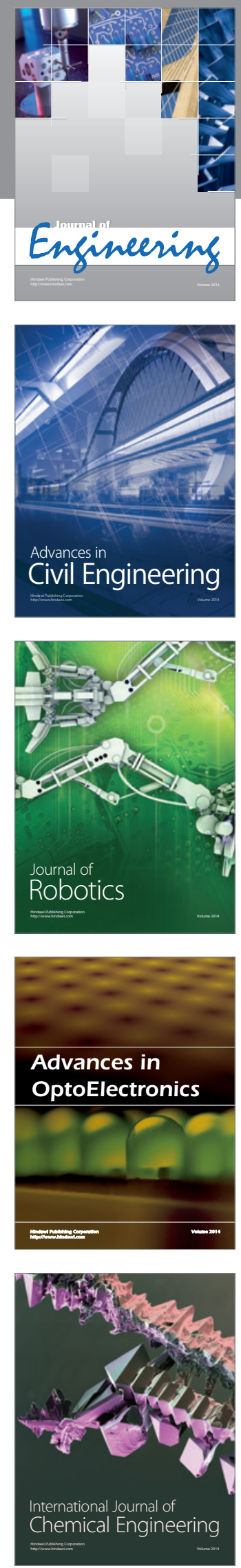

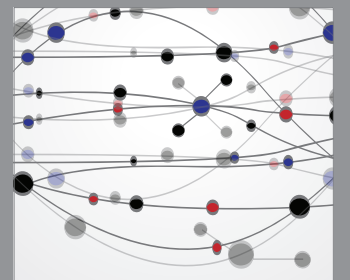

The Scientific World Journal
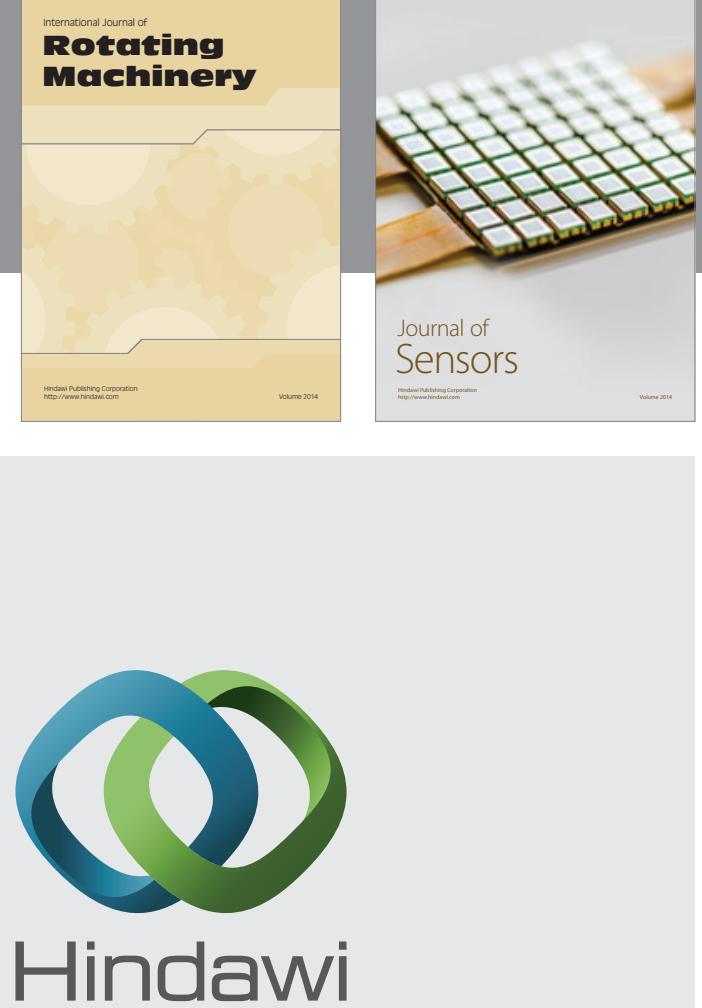

Submit your manuscripts at http://www.hindawi.com
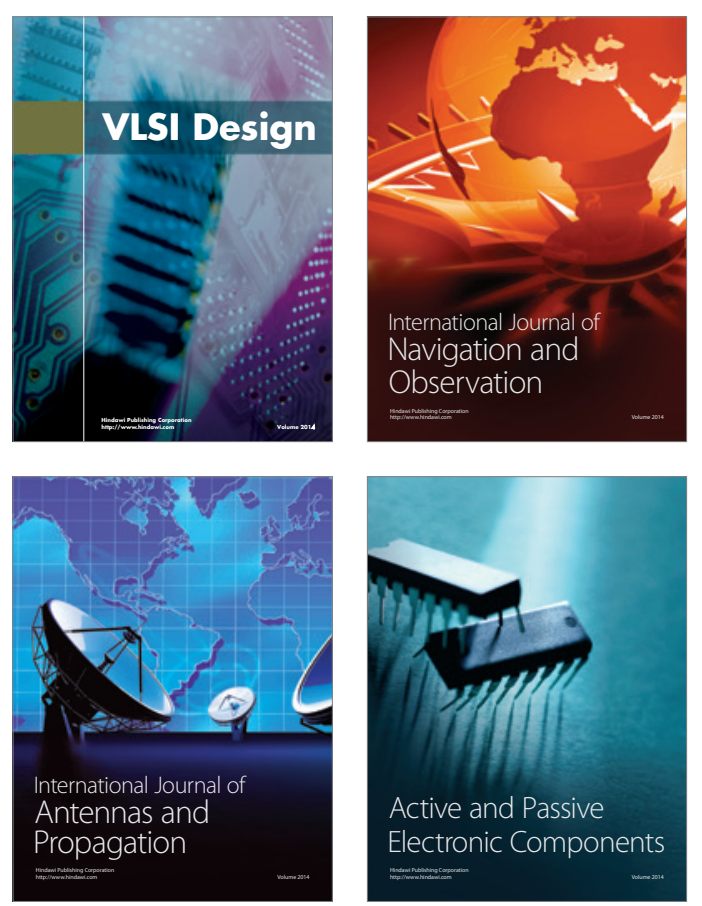
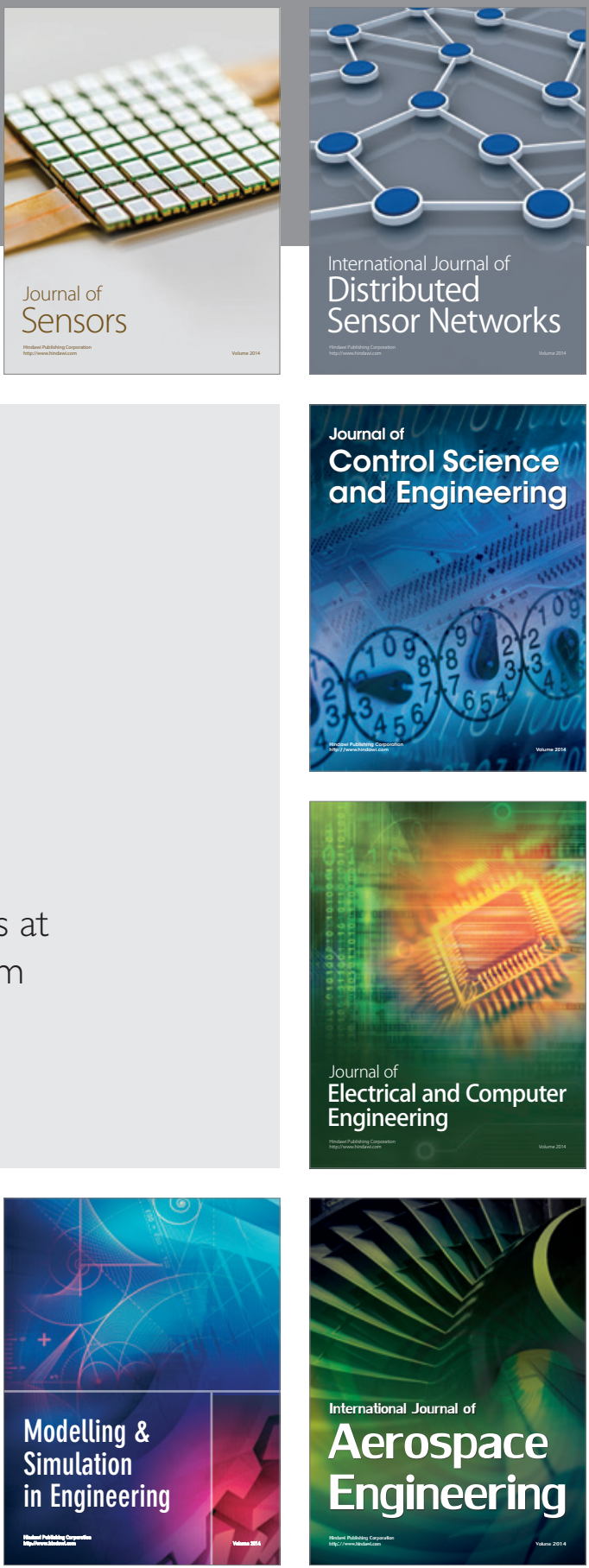

Journal of

Control Science

and Engineering
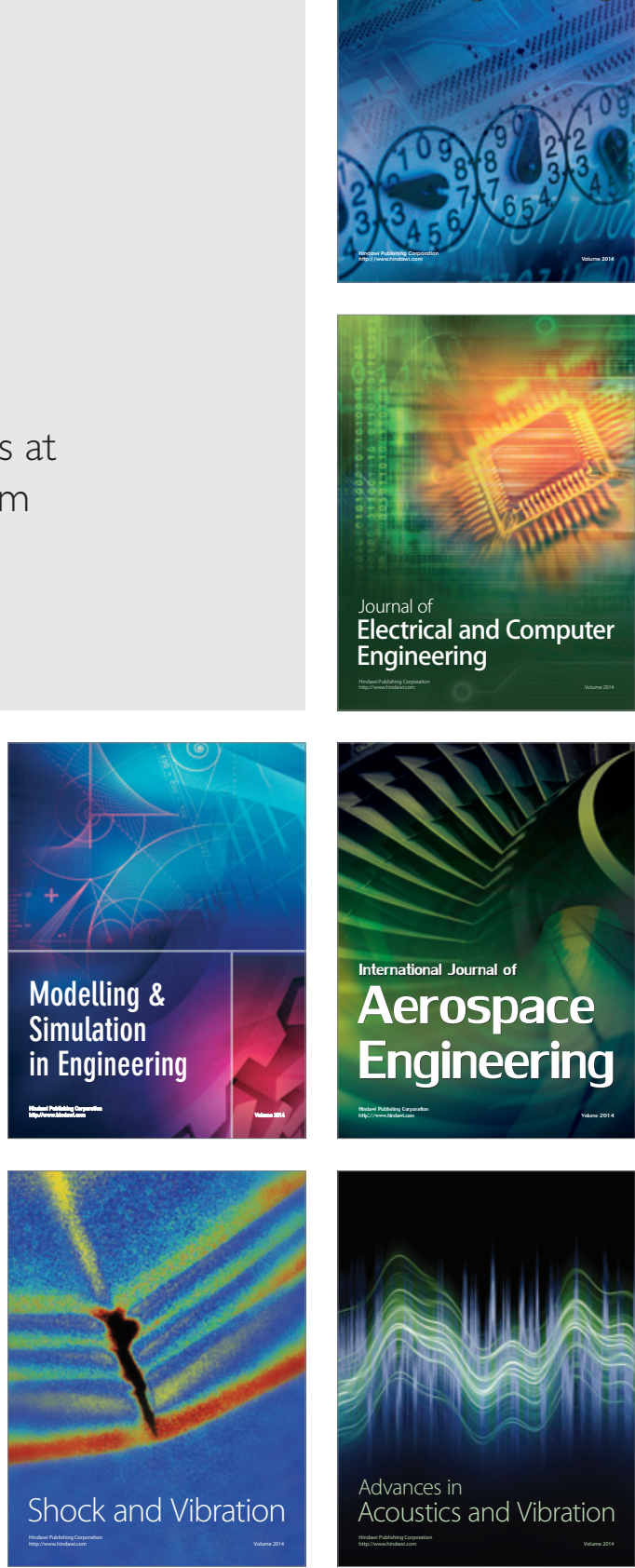